\title{
Survey of ERG expression in normal bone marrow and myeloid neoplasms
}

\author{
Nicholas J. Olson ${ }^{1} \cdot$ Deborah L. Ornstein ${ }^{1} \cdot$ Konstantinos Linos ${ }^{1}$ (i) \\ Received: 16 July 2019 / Accepted: 15 October 2019 / Published online: 15 November 2019 \\ (C) Springer-Verlag GmbH Germany, part of Springer Nature 2019
}

\begin{abstract}
The immunohistochemical stain ERG is a useful diagnostic marker for leukemia cutis. Translocations involving the $E R G$ gene have been identified in acute myeloid leukemia (AML) and it plays critical roles in differentiation of hematopoietic stem cells. However, little is known about ERG expression in the bone marrow or in myeloid neoplasms. The aim of this study is to characterize ERG expression in normal bone marrow specimens, and those with various myeloid neoplasms. We performed immunohistochemical studies assessing ERG expression in bone marrow biopsies obtained over a 1-year period, in which myeloperoxidase (MPO) was used to assess granulocyte populations. Twenty-eight bone marrow biopsies (6 normal, 12 with acute myeloid leukemia (AML), 6 with myeloproliferative neoplasms (MPN), and 4 with myelodysplastic/MPN) were identified. Strong nuclear ERG staining was present within the granulocytes and precursors in near complete concordance with MPO in 26/ $28(93 \%)$ cases. Fifty-eight percent of AML cases showed more staining for ERG than MPO in the leukemic cells. ERG can be useful for assessing granulocyte populations in bone marrow biopsies, and in many instances of AML, stained a proportion of myeloblasts.
\end{abstract}

Keywords ERG $\cdot$ Myeloid neoplasms $\cdot$ Bone marrow $\cdot$ Acute myeloid leukemia

\section{Introduction}

E26 transformation-specific (ETS) regulated gene-1 (ERG) is a member of the ETS family of transcription factors on chromosome 21 [1]. ERG has several biological roles in cell proliferation, differentiation, angiogenesis, inflammation, and apoptosis [2]. In vascular endothelial cells, $E R G$ plays a role in regulating angiogenesis and cell apoptosis [3]. Additionally, $E R G$ controls the $\mathrm{Wnt} / \beta$-catenin pathway by increasing $\beta$ catenin stability, and deletion of $E R G$ in endothelial cells causes defective angiogenesis and decreased vascular stability [4]. In chondrocytes, $E R G$ also has a regulatory role in development and maintenance of the cells $[5,6]$. In hematopoiesis,

This material was presented in poster form at the United States and Canadian Academy of Pathology Annual Meeting. National Harbor, MD. 3/18/2019

Konstantinos Linos

Konstantinos.Linos@hitchcock.org

1 Department of Pathology and Laboratory Medicine, Dartmouth-Hitchcock Medical Center, Geisel School of Medicine at Dartmouth, 1 Medical Center Drive, Lebanon, NH 03756, USA studies have shown that $E R G$ plays a critical role in the development/function of hematopoietic stem cells and regulates their self-renewal and differentiation $[1,7]$.

In tumorigenesis, $E R G$ appears to be a potent oncogene and has been implicated in the development of numerous tumors including prostatic adenocarcinoma [8] and Ewing sarcoma [9]. Additionally, in approximately $1 \%$ of acute myeloid leukemias (AML), ERG is fused with FUS resulting in the translocation $\mathrm{t}(16 ; 21)[10,11]$. AML with $\mathrm{t}(16 ; 21)$ can demonstrate hemophagocytosis in the leukemic cells, and has been associated with adverse prognosis including low rates of remission following induction chemotherapy, high relapse rates, and increased mortality [11]. The development of an anti-ERG Immunohistochemical (IHC) stain, also called ERG, has been used in surgical pathology to identify prostate adenocarcinoma [12] as well as sarcomas with translocations involving $E R G$ [9]. Moreover, ERG has also been shown to be a lineage-specific marker, most prominently used to identify tumors with endothelial differentiation $[12,13]$. Other tumors that express ERG include chondrosarcoma [14], chondromyxoid fibroma [14], chondroblastic osteosarcoma [14], clear cell chondrosarcoma [14], and phosphaturic mesenchymal tumor [15]. The ERG immunostain has also been 
shown to be quite useful in leukemia cutis, distinguishing it from reactive myeloid infiltrates in the skin [2].

Recently, while using ERG during evaluation of a case of prostatic adenocarcinoma metastatic to the spine, we noted that the normal myeloid components of the bone marrow stained strongly positive for ERG. Despite an apparent link between $E R G$ and myeloid development/function, there is limited information about ERG expression in medullary myeloid neoplasms [2, 12]. To date, no studies have assessed ERG expression in normal bone marrow or in bone marrows involved by myeloid neoplasms. Therefore, the aim of this study was to characterize ERG expression in normal bone marrow biopsy specimens and in those involved by various myeloid neoplasms. The staining for ERG was then compared to staining for myeloperoxidase (MPO), which is frequently used to assess granulocyte populations in bone marrow biopsies $[16,17]$.

\section{Materials and methods}

A protocol involving acquisition of archival bone marrow biopsy specimens, clinical data, and subsequent tissue analysis was approved by the Committee for the Protection of Human Subjects at our institution. The search criteria included all trephine bone marrow biopsies performed over a one-year period in which MPO immunohistochemistry (pre-diluted, Leica Biosystems, Buffalo Grove, IL) was utilized to assess the granulocytic population. If performed, the results of cytogenetic analysis and the results of a 54-gene next-generation sequencing myeloid mutations panel (TruSight Myeloid Panel; Illumina Inc., San Diego, CA) were also collected. Additional stains that were performed to assess other bone marrow lineages, such as glycophorin for erythroid elements, CD61 for megakaryocytes, and CD34 for blasts, were also reviewed.

All biopsies were formalin fixed, decalcified in a formic acid solution for $90 \mathrm{~min}$, and embedded in paraffin. The formalin-fixed, paraffin-embedded (FFPE) tissue sections were stained with hematoxylin and eosin (H\&E) using routine laboratory protocols. Immunohistochemical studies were performed using the Leica BOND-III (Leica Biosystems, Buffalo Grove, IL) autostainer. ERG immunohistochemistry was performed on all slides using a rabbit monoclonal antibody specific to the C-terminus (ab133264, 1:250 dilution; Abcam, Cambridge, MA) using the same Leica BOND-III autostainer. Antigen retrieval for ERG was done using heatinduced epitope retrieval solution 1 (ER-1) (Leica Biosystems, Buffalo Grove, IL) for $40 \mathrm{~min}$ at $100{ }^{\circ} \mathrm{C}$, and antigen retrieval for MPO is performed at our institution using heat-induced epitope retrieval solution 2 (ER-2) (Leica Biosystems, Buffalo Grove, IL) for $10 \mathrm{~min}$. Nuclear staining intensity was graded as 0 (absent staining), $1+($ minimal staining), $2+$ (staining less than native endothelial cells), and $3+$ (staining equal to native endothelial cells). The percentage of positive cells was then compared with previously performed MPO immunohistochemistry. The correlation of ERG-positive cells to MPO positive cells was scored on a scale of $0-3$, which was defined as 0 (no correlation), $1+$ $(0-25 \%$ correlation $), 2+(25-75 \%$ correlation $)$, and $3+(>$ $75 \%$ correlation).

\section{Results}

Our search identified 28 cases meeting criteria for inclusion in this study. These included six normal bone marrow biopsy specimens, 12 biopsy specimens involved by acute myeloid leukemia (AML), six by myeloproliferative neoplasms (MPN), and four by myelodysplastic/myeloproliferative neoplasms (MDS/MPN) (Table 1). ERG staining was interpretable in all 28 cases $(100 \%)$. In most instances, the nuclear staining was strong, diffuse, and uniform throughout the sample.

We first examined the normal bone marrow biopsy specimens. All six normal specimens showed strong nuclear positivity for ERG in the granulocytes and precursors with near $100 \%$ concordance with MPO (Fig. 1). Although the ERG staining intensity was strong $(3+$ intensity) in the majority of granulocytes, several cases showed slightly diminished intensity $(1+$ to $2+)$ in band forms and segmented neutrophils, similarly to the pattern we observed with MPO. The erythroid precursors were always negative for ERG, which was confirmed by glycophorin IHC in most cases. The megakaryocytes were also negative for ERG (Fig. 1h).

Next, in 11 of $12(92 \%)$ specimens involved by AML, there was $3+$ concordance between ERG and MPO staining (Fig. 2). One patient, case 15 , showed $2+$ concordance with decreased $(2+)$ ERG staining intensity; however, all remaining cases of AML showed strong $(3+)$ ERG staining intensity. In fact, several cases in which MPO staining was weak demonstrated strong, diffuse, nuclear staining for ERG (Fig. 2e, f). Additionally, in 7 of $12(58 \%)$ AML cases (cases 7, 8, 9, 10, 13,16 , and 18), the ERG stain identified a larger proportion of the leukemic cells than the MPO stain. In cases 12 and 14, although ERG staining was 100\% concordant with MPO, both stained less than $10 \%$ of leukemic cells (Fig. 2h, i).

Among the six patients with MPNs, there were four cases of primary myelofibrosis (PMF) and one case each of chronic myeloid leukemia (CML), and polycythemia vera (PV) (Fig. 3). In 5 of 6 (83\%) myeloproliferative neoplasms, there was 3 + concordance between ERG and MPO, with one case (case 24) of PMF showing $2+$ concordance. All staining was strong ( $3+$ intensity), nuclear, and again limited to the granulocytic cell lines. One other case of PMF demonstrated slightly 


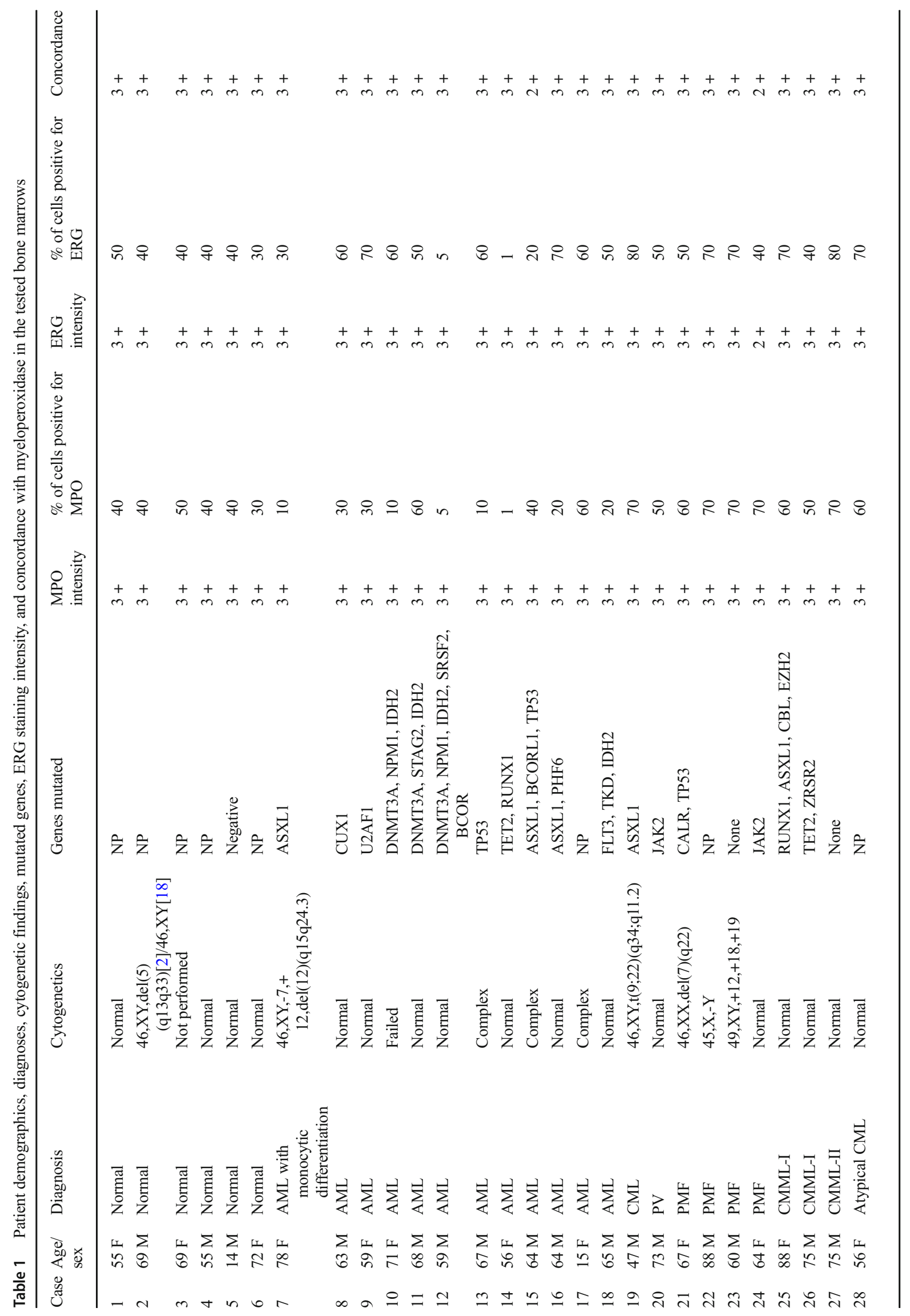




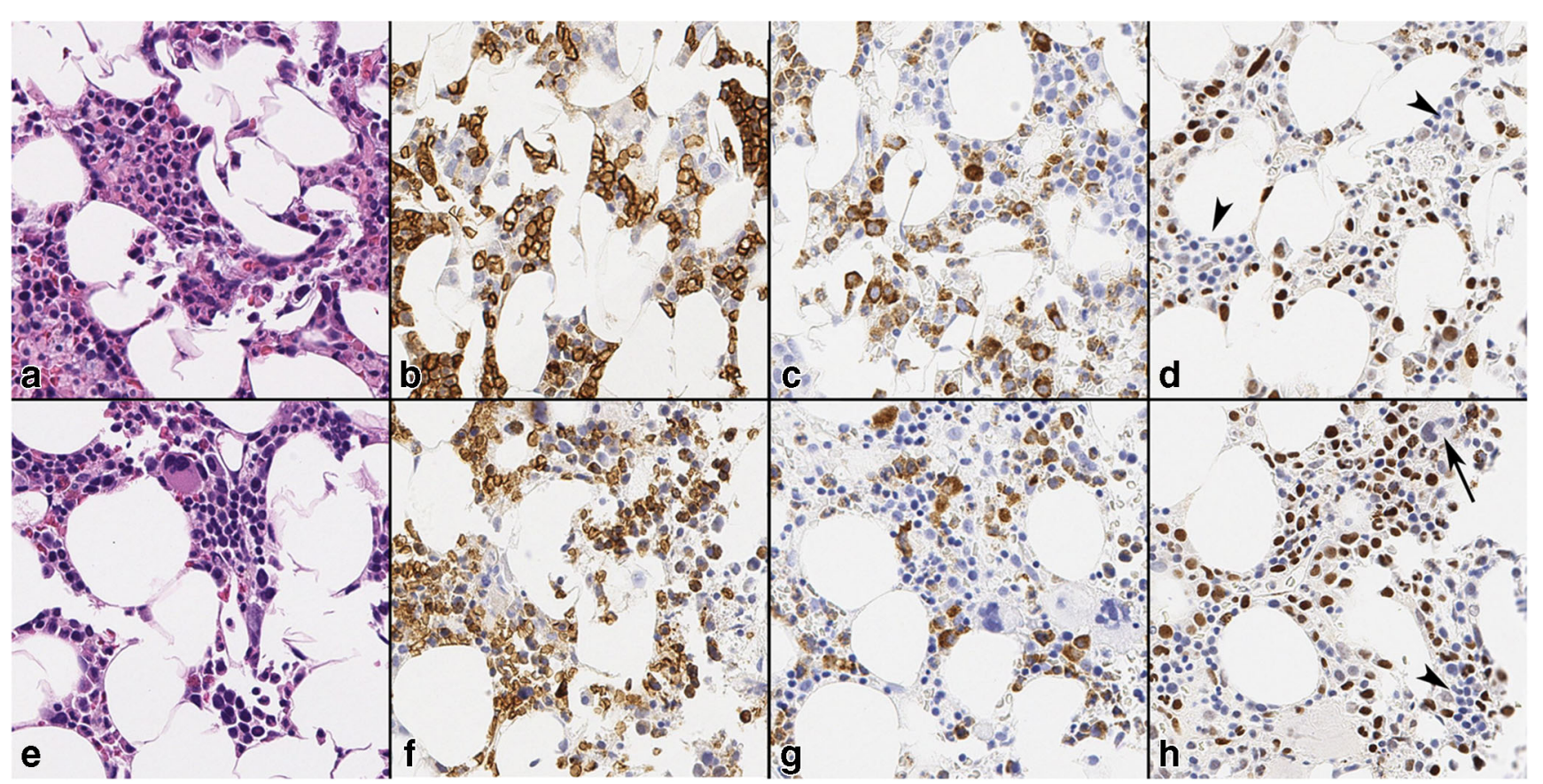

Fig. 1 Normal bone marrow biopsy specimens. a-d Case 3. e-h Case 4. a, e H\&E stains. b, f Glycophorin immunostain, highlighting nests of erythroid precursors. c, $\mathbf{g}$ MPO immunostain, highlighting granulocytes and precursors. $\mathbf{d}, \mathbf{h}$ ERG staining the same granulocytes and precursors

patchy staining compared to MPO, but the overall concordance was still $3+$.

There were four patients with MDS/MPN, including two patients with chronic myelomonocytic leukemia (CMML)-1, one patient with CMML-2, and one patient with atypical CML. All four biopsies showed strong $(3+$ intensity) nuclear staining for ERG in the granulocytes, precursors, and rare monocytes in near $100 \%$ concordance with MPO.

\section{Discussion}

In surgical pathology, ERG has proven to be useful as a lineage-specific marker in diagnosing benign and malignant vascular tumors, as well as identifying tumors with fusions involving $E R G$ such as prostatic adenocarcinoma which approximately $50 \%$ harbor a TMPRSS2-ERG fusion $[8,12,13]$. ERG was extensively studied by Miettinen et al. [12] where it was noted to be expressed in a subpopulation of myeloid cells and in 7 of 10 extramedullary myeloid tumors examined. Unfortunately, however, further characterization of the ERGpositive myeloid cells was not performed in that study. Xu et al. [2] examined 16 cases of leukemia cutis and found ERG expression in $13(81.4 \%)$ cases but in none of the reactive conditions. The authors concluded that ERG positivity was a strong and useful tool in distinguishing leukemia cutis from benign and reactive myeloid infiltrates. However, until now, there has been limited data on ERG expression in normal as the MPO immunostain. Note the strong nuclear staining for ERG in all forms, and negative staining in megakaryocytes (arrow) and erythroid islands (arrowheads). All magnifications are $\times 400$

bone marrow or in myeloid neoplasms involving the bone marrow.

This study represents the first dataset examining the expression patterns of ERG in normal bone marrow, and in those involved by various myeloid neoplasms. Our study examined normal bone marrow as well as several conditions with aberrant myeloid maturation that often require assessment of the various myeloid populations including AML, CML, PMF, PV, and CMML. Currently, the most commonly used stains for identifying the granulocyte/myeloid population include MPO, lysozyme, CD13, CD33, and CD43 [18, 19]. MPO is a cytoplasmic stain that marks myeloperoxidase, a major component of the primary granules of neutrophils and eosinophils, and both cell types show strong reactivity [16]. Occasionally, cells of the monocytic cell line can be weakly positive, while mast cells, lymphocytes, erythroid precursors, and megakaryocytes are negative [18]. At our institution, MPO is the most frequently used stain to assess granulocyte populations, as it has been shown to be the most specific [18].

In contrast to the other granulocytic lineage markers, ERG offers the benefit of being a nuclear stain, and in our cases, we observed considerably less background staining compared to MPO. This has also been noted by others when ERG was compared to CD31 and CD34 as an endothelial marker [12, 13]. Both CD31 and CD34 have a membranous staining pattern and when they were compared to ERG, which is a nuclear stain, ERG was observed to be more specific and easier to interpret. We noted a slight decrease in ERG staining intensity 


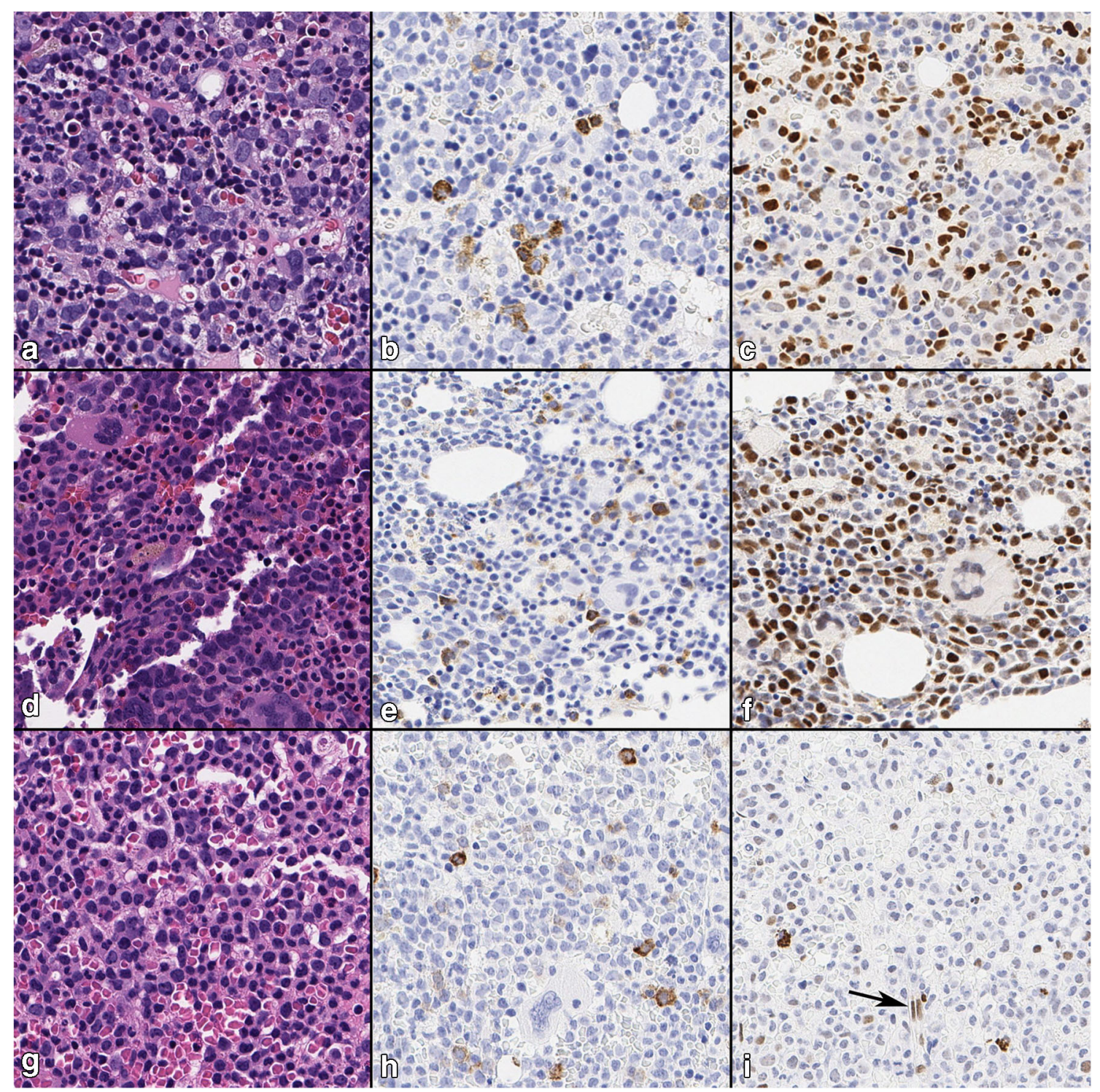

Fig. 2 Acute myeloid leukemia. a-c Case 13. H\&E, MPO, and ERG, respectively. In this case, the MPO stain showed faint cytoplasmic staining in many of the blasts while ERG showed strong diffuse nuclear staining. d-f Case 7. H\&E, MPO, and ERG, respectively. Again MPO weakly highlighted the granulocytes while ERG was diffusely positive.

in a minority of the band forms and more mature neutrophils, which was also reflected in our MPO stain. A similar finding of decreased ERG expression was also observed when leukemia cutis samples were compared to reactive leukocytic infiltrates by $\mathrm{Xu}$ et al. [2]. Although all 16 of the "reactive leukocytic infiltrates" described by the authors were considered negative for ERG, they in fact describe $1+$ staining in 13 of the 16 cases. In their study, only $2+$ and $3+$ staining was considered positive; thus, all the reactive infiltrates were designated as negative for ERG. Our observation of decreased ERG staining in some of our cases appears in line with theirs. However, ERG was strongly expressed ( $3+$ intensity) in mature neutrophils in the majority of our cases, including all of
Note negative staining islands of erythroid precursors. g-i Case 14. H\&E, MPO, and ERG, respectively. Some cases of AML had minimal ERG staining in leukemic cells, but remained concordant with myeloperoxidase. Note positive internal control endothelial cells (Arrow). All magnifications are $\times 400$

the normal bone marrows (Fig. 1d, h). Therefore, this may reflect downregulation of ERG as part of normal granulocyte maturation. For example, the more immature neutrophils present within the bone marrow are more likely to strongly express ERG, and once neutrophils migrate to the peripheral circulation, ERG expression is weak to absent similar to the observation by $\mathrm{Xu}$ et al. [2]. However, further studies are required to elucidate the mechanism underlying this finding.

In 7 of 12 cases of AML, ERG staining showed concordance with MPO, and appeared to stain a higher proportion of cells including a subset of the blasts. The cytogenetic and molecular data did not indicate a common mutated gene within this group to explain this finding. In fact, the range of 


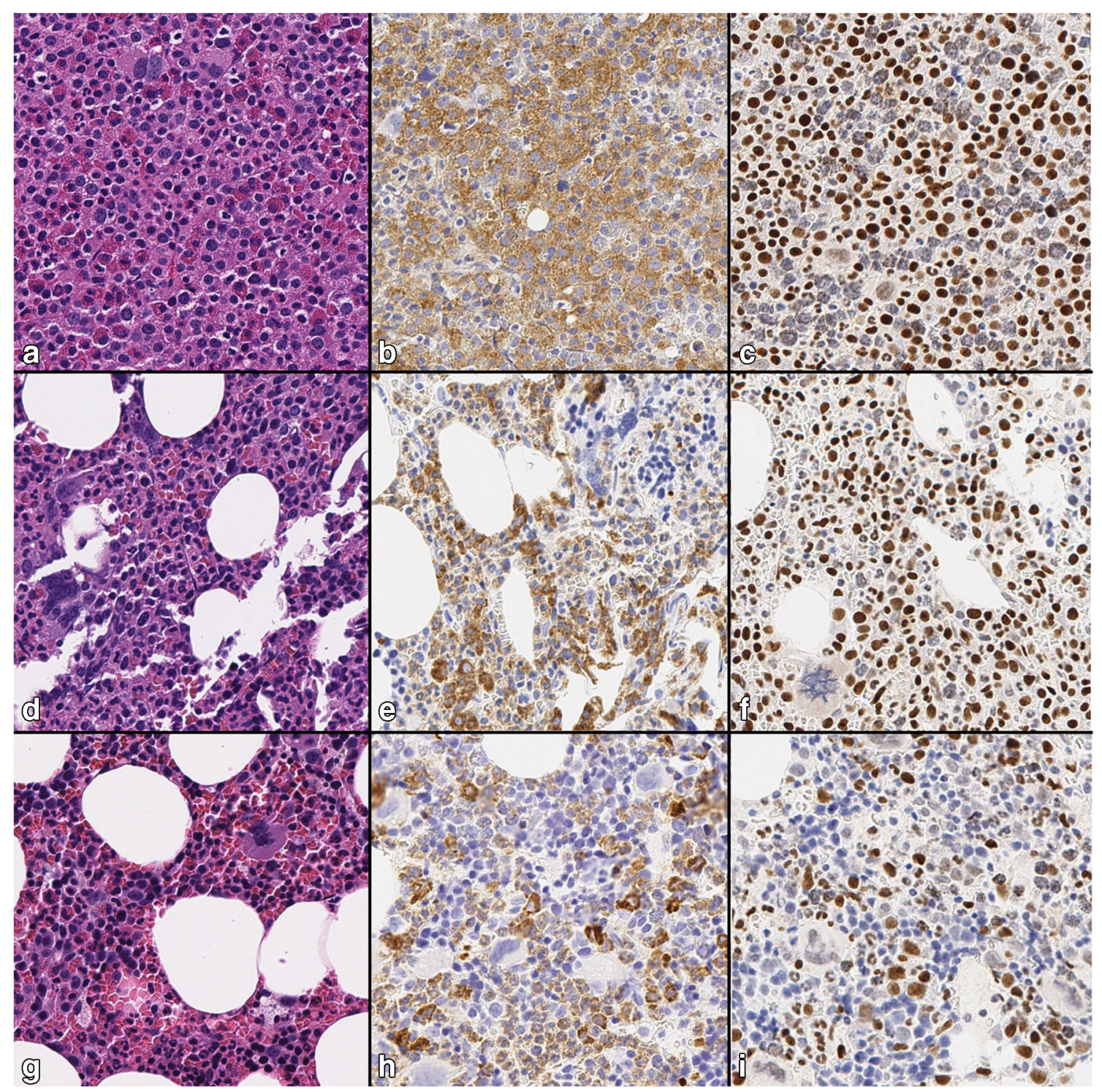

Fig. 3 Myeloproliferative neoplasms. a-c CML, case 19. a H\&E showing dense infiltration of the marrow by micromegakaryocytes, eosinophils, and myeloid precursors. b The granulocytes showed strong cytoplasmic positivity for MPO. c ERG highlighting the same cell population with strong nuclear staining. $\mathbf{d}-\mathbf{f}$ Primary myelofibrosis, case 23. d H\&E showing marked megakaryocytic atypia. e, f MPO and

genetic aberrations was quite broad in this small group, with three cases demonstrating complex karyotypes, 2 harboring normal karyotypes, whereas the last case failed karyotyping. It is possible that weak staining with our in-house MPO stain made it appear that ERG was staining a higher proportion of cells by contrast. Since MPO staining was performed at the time of the original bone marrow assessment, this opens up the possibility of error (e.g., technical issues at the time of staining, stain viability loss over time). However, the cases with increased ERG staining compared to MPO span a large time range with a proportion of cases showing equal staining for ERG and MPO. Accordingly, we believe that the increased
ERG, respectively, highlighting the granulocytes while the megakaryocytes and erythroid precursors were negative. g-i Polycythemia vera, case 21 . g H\&E showing erythroid hyperplasia. h, I MPO and ERG, respectively, highlighting granulocytes. All magnifications $\times 400$

ERG staining observed in a subset of cases likely represents true increased ERG expression compared to MPO.

The specificity of ERG for granulocytes is unknown in hematolymphoid neoplasms. In the large study by Miettinen et al. [12], ERG expression was examined in seven cases of anaplastic large cell lymphoma, nine cases of diffuse large Bcell lymphoma, nine cases of mantle cell lymphoma, and seven cases of T-lymphoblastic lymphoma (T-ALL). None of these cases showed nuclear expression of ERG. However, $E R G$ transcriptional deregulation has been described in a subset of B-lymphoblastic leukemia/lymphoma (B-ALL) [20]. Another study found that patients with acute lymphoblastic 
leukemia/lymphoma had higher expression of ERG than normal controls, and that lower levels of ERG expression correlated with a T-ALL phenotype and an inferior relapse-free survival rate [21]. However, these studies used gene expression profiling examining DNA copy number alterations by SNP microarrays, and reverse transcription polymerase chain reaction (RT-PCR), respectively, and did not examine ERG expression by IHC analysis. Additionally, a recent immunohistochemical analysis of 12 multiple myeloma specimens by Knief et al. found strong nuclear ERG expression in $>90 \%$ of cells in all 12 cases [22]. Moreover, a study comparing mouse and rabbit anti-ERG monoclonal antibodies in lymph nodes with metastatic prostate carcinoma found that follicular lymphocytes and sinus histiocytes stained positively when a rabbit anti-ERG monoclonal antibody was used but they were negative when a mouse monoclonal antibody was used [23]. As mentioned previously, the antibody used in our study was a rabbit monoclonal antibody and we did not identify any lymphocyte staining with ERG. Therefore, the early evidence indicates that ERG does have potential pitfalls, and it may not be a lineage-specific marker for granulocytes. However, more studies examining ERG expression in other hematolymphoid neoplasms are needed to properly assess the sensitivity and specificity of ERG for the granulocyte cell line.

In summary, we show that ERG is a useful lineage marker for assessing granulocyte populations in bone marrow biopsy specimens. ERG highlights granulocytes and precursors in concordance with MPO, and, in cases of AML, ERG may be an improvement over MPO as it seems to highlight a larger proportion of the abnormal cell population with considerably less background staining. ERG IHC therefore offers hematopathologists a nuclear stain with lower background staining to improve identification of granulocytic populations in normal and diseased bone marrow biopsy specimens.

\section{Compliance with ethical standards}

A protocol involving acquisition of archival bone marrow biopsy specimens, clinical data, and subsequent tissue analysis was approved by the Committee for the Protection of Human Subjects at our institution.

Conflict of interest The authors declare they have no conflict of interest.

\section{References}

1. Loughran SJ, Kruse EA, Hacking DF, de Graaf CA, Hyland CD, Willson TA, Henley KJ, Ellis S, Voss AK, Metcalf D, Hilton DJ, Alexander WS, Kile BT (2008) The transcription factor Erg is essential for definitive hematopoiesis and the function of adult hematopoietic stem cells. Nat Immunol 9:810-819

2. Xu B, Naughton D, Busam K, Pulitzer M (2016) ERG is a useful immunohistochemical marker to distinguish leukemia cutis from nonneoplastic leukocytic infiltrates in the skin. Am J Dermatopathol 38:672-677
3. Birdsey GM, Dryden NH, Amsellem V et al (2008) Transcription factor Erg regulates angiogenesis and endothelial apoptosis through VE-cadherin. Blood 111:3498-3506

4. Birdsey GM, Shah AV, Dufton N, Reynolds LE, Osuna Almagro L, Yang Y, Aspalter IM, Khan ST, Mason JC, Dejana E, Göttgens B, Hodivala-Dilke K, Gerhardt H, Adams RH, Randi AM (2015) The endothelial transcription factor ERG promotes vascular stability and growth through Wnt/ $\beta$-catenin signaling. Dev Cell 32:82-96

5. Iwamoto M, Higuchi Y, Enomoto-Iwamoto M et al (2001) The role of ERG (ets related gene) in cartilage development. Osteoarthr Cartil 9(Suppl A):S41-S47

6. Iwamoto M, Tamamura Y, Koyama E et al (2007) Transcription factor ERG and joint and articular cartilage formation during mouse limb and spine skeletogenesis. Dev Biol 305:40-51

7. Knudsen KJ, Rehn M, Hasemann MS, Rapin N, Bagger FO, Ohlsson E, Willer A, Frank AK, Søndergaard E, Jendholm J, Thorén L, Lee J, Rak J, Theilgaard-Mönch K, Porse BT (2015) ERG promotes the maintenance of hematopoietic stem cells by restricting their differentiation. Genes Dev 29:1915-1929

8. Mosquera J-M, Mehra R, Regan MM, Perner S, Genega EM, Bueti G, Shah RB, Gaston S, Tomlins SA, Wei JT, Kearney MC, Johnson LA, Tang JM, Chinnaiyan AM, Rubin MA, Sanda MG (2009) Prevalence of TMPRSS2-ERG fusion prostate cancer among men undergoing prostate biopsy in the United States. Clin Cancer Res $15: 4706-4711$

9. Wang W-L, Patel NR, Caragea M, Hogendoorn PC, López-Terrada D, Hornick JL, Lazar AJ (2012) Expression of ERG, an Ets family transcription factor, identifies ERG-rearranged Ewing sarcoma. Mod Pathol 25:1378-1383

10. Kong XT, Ida K, Ichikawa H, Shimizu K, Ohki M, Maseki N, Kaneko Y, Sako M, Kobayashi Y, Tojou A, Miura I, Kakuda H, Funabiki T, Horibe K, Hamaguchi H, Akiyama Y, Bessho F, Yanagisawa M, Hayashi Y (1997) Consistent detection of TLS/ FUS-ERG chimeric transcripts in acute myeloid leukemia with $\mathrm{t}(16 ; 21)(\mathrm{p} 11 ; \mathrm{q} 22)$ and identification of a novel transcript. Blood 90:1192-1199

11. Jekarl DW, Kim M, Lim J, Kim Y, Han K, Lee AW, Kim HJ, Min WS (2010) CD56 antigen expression and hemophagocytosis of leukemic cells in acute myeloid leukemia with $\mathrm{t}(16 ; 21)(\mathrm{p} 11 ; \mathrm{q} 22)$. Int J Hematol 92:306-313

12. Miettinen M, Wang Z-F, Paetau A, Tan SH, Dobi A, Srivastava S, Sesterhenn I (2011) ERG transcription factor as an immunohistochemical marker for vascular endothelial tumors and prostatic carcinoma. Am J Surg Pathol 35:432-441

13. McKay KM, Doyle LA, Lazar AJ, Hornick JL (2012) Expression of ERG, an Ets family transcription factor, distinguishes cutaneous angiosarcoma from histological mimics. Histopathology 61:989991

14. Shon W, Folpe AL, Fritchie KJ (2015) ERG expression in chondrogenic bone and soft tissue tumours. J Clin Pathol 68:125129

15. Tajima S, Takashi Y, Ito N, Fukumoto S, Fukuyama M (2016) ERG and FLI1 are useful immunohistochemical markers in phosphaturic mesenchymal tumors. Med Mol Morphol 49:203-209

16. Pinkus GS, Pinkus JL (1991) Myeloperoxidase: a specific marker for myeloid cells in paraffin sections. Mod Pathol 4:733-741

17. Dunphy CH, Polski JM, Evans HL et al (2001) Evaluation of bone marrow specimens with acute myelogenous leukemia for CD34, CD15, CD117, and myeloperoxidase. Arch Pathol Lab Med 125: 1063-1069

18. Dabbs D (2002) Diagnostic immunohistochemistry, 5th edn. Elsevier Health Sciences, Philadelphia

19. Dixon J, Kaklamanis L, Turley H, Hickson ID, Leek RD, Harris AL, Gatter KC (1994) Expression of aminopeptidase-n (CD 13) in normal tissues and malignant neoplasms of epithelial and lymphoid origin. J Clin Pathol 47:43-47 
20. Zhang J, McCastlain K, Yoshihara H et al (2016) Deregulation Of DUX4 And ERG in acute lymphoblastic leukemia. Nat Genet 48: 1481-1489

21. Zhao H-Z, Jia M, Luo Z-B, Xu XJ, Li SS, Zhang JY, Guo XP, Tang YM (2017) ETS-related gene is a novel prognostic factor in childhood acute lymphoblastic leukemia. Oncol Lett 13:455-462

22. Knief J, Reddemann K, Gliemroth J et al (2017) ERG expression in multiple myeloma - a potential diagnostic pitfall. Pathol Res Pract 213:130-132
23. Braun M, Goltz D, Shaikhibrahim Z, Vogel W, Böhm D, Scheble V, Sotlar K, Fend F, Tan SH, Dobi A, Kristiansen G, Wernert N, Perner S (2012) ERG protein expression and genomic rearrangement status in primary and metastatic prostate cancer-a comparative study of two monoclonal antibodies. Prostate Cancer Prostatic Dis 15: $165-169$

Publisher's note Springer Nature remains neutral with regard to jurisdictional claims in published maps and institutional affiliations. 